\title{
3D Seismic Acquisition Technology and Effect in HX Volcanic Area in Liaohe Depression
}

\author{
Haibo Wang1,2, Bing Liu², Qiwei Zou3, Lizhi Sun², Wei Zhang², Jiantao Tian², Meibin Tang² \\ ${ }^{1}$ School of Geophysics and Information Technology, China University of Geosciences, Beijing, China \\ ${ }^{2}$ BGP Inc., CNPC, Panjin, China \\ ${ }^{3}$ Liaohe Oil Field, CNPC, Panjin, China \\ Email: wanghaibo004@qq.com
}

Received 28 June 2016; accepted 17 July 2016; published 20 July 2016

Copyright (C) 2016 by authors and Scientific Research Publishing Inc.

This work is licensed under the Creative Commons Attribution International License (CC BY).

http://creativecommons.org/licenses/by/4.0/

(c) (i) Open Access

\begin{abstract}
Multi-stages volcanic are available in $\mathrm{HX}$ area, shielding the seismic waves. Previous seismic acquisitions of large size bin, less fold coverage and narrow azimuth result in indistinct fault images, low $\mathrm{S} / \mathrm{N}$ ratio and the difficulty of multi-stages volcanic characterization. In reference to the successful experience of domestic and overseas volcanic exploration, the low frequency excitation and receiving, and survey with wide range, high coverage, wide azimuth should be paid more attention, associated with two-dimensional and three-dimensional wave equation forward modeling and real data processing contrast analysis method. The image of underlying strata and fault are remarkably improved in the new method, according to the processing results of new seismic data. The new method will provide technical reference for the similar volcanic development area in the future seismic acquisition design.
\end{abstract}

\section{Keywords}

Volcanic, 3D Seismic, Low Frequency, Wide Azimuth, High Fold, Small Bin Size

\section{Introduction}

HX area is abundant of volcanic in Liaohe depression, structurally located at the south central of eastern sag of Liaohe depression. HX area developed several sets of volcanic from Es3, Es1 and Ed in the longitudinal direction, the volcanic of different layers superimposed in the plane direction, and its uneven distribution. The volcanic in this area gives priority to the overflow facies; seismic reflection characteristics are layered, wedge reflection with strong amplitude. The volcanic and sedimentary rock superimposed each other, the volcanic has a 
strong shielding effect on the underlying formation; it also brings multiple wave problems, resulting in indistinct fault images, low $\mathrm{S} / \mathrm{N}$ ratio and the difficulty of multi-stages volcanic characterization. In recent years, many wells in eastern sag obtain high-yield industrial oil in volcanic reservoir, but the poor quality of seismic data constrained exploration and development process.

Volcanic exploration is a difficult in the field of exploration at home and abroad. On seismic acquisition, we mainly used forward modeling and illumination technology to help study seismic wave field and energy distribution, the wide angle seismic observation, observation of converted wave and survey of high density and wide azimuth. Scholars at home and abroad carry out an in-depth research on volcanic acquisition. It is especially concerned to use the wide angle seismic technology abroad [1]-[4]. Purnellg [5] and Moshe [6] discussed to improve the imaging quality of deep basalt area by using the converted wave. Ziolkowski [7] introduced the imaging of the volcanic with the low frequency information. Hu Zhongping [8] analyzed the characteristics of the wide angle seismic signal and the effect of the wide angle reflection for noise by the forward theoretical model, guided the actual wide angle seismic data processing and achieved sub basalt seismic imaging. An Xueyong [9] and Zhao Jianzhang [10] put forward the suggestion of seismic acquisition survey parameters in view of the problems of the development of the surface layer and the superficial layer. She Deping [11] [12] using wave equation wave field numeric simulation technique, showed the low frequency signals having strong capacity both penetrating thin shielding high velocity basalt and reducing diffraction noises produced from rough surface, improving the imaging quality of deep beds below the shielding layer through using low frequency. Zhang Guangde [13] introduced geometry optimization using 3-D Gaussian beam forward modeling theory. The above described in the literature of seismic acquisition didn't achieve high density sampling and wide azimuth acquisition, which cannot solve the problem of volcanic layer and the underlying strata imaging well. According to the seismic exploration practice in recent years, high density and wide azimuth seismic acquisition are the effective means to solve the problem of complex geological.

Survey with huge offset, wide-angle reflection and higher folds with 175 times (aspect ratio is only 0.28) is used in HX area, but the problems of volcanic strata and the fault imaging can't be solved well. By synthesizing the volcanic exploration of domestic and overseas and successful experience of "Two Wide and One High" (Wide Azimuth, Wide Frequency and High Density) researches in recent years [14]. The 2D and 3D wave equation forward modeling are carried out. By analyzing and processing actual data of HX region, the wide azimuth purpose is put forward (4000 $\mathrm{m}$ target layer to be full azimuth), high fold, small bin size, big offset survey, using low frequency vibrator to achieve broad band shooting, and realize "Two Wide and One High" acquisition. Through data processing and interpretation, we achieve good results which effectively improve the imaging of underlying strata and fracture.

\section{Design Survey Aim at Volcanic Imaging}

Based on the development of volcanic of this area, conducting acquisition survey design, conducting forward modeling research using different frequency wavelet, analyzing the impact of different bin, azimuth, folds and offset on the data quality.

\subsection{Response of Volcanics to Different Shooting Frequencies}

According to the previous seismic data and well data, building model containing multi-stages of thin layer volcanic (Figure 1(a), 4 sets of volcanic layer are set up according to the comprehensive logging diagram). Conducting forward modeling using different dominant frequency. Figures 1(b)-(e) are single shot record and profile with shooting frequency $9 \mathrm{~Hz}$ and $30 \mathrm{~Hz}$. As for the energy and continuity under the volcanic (Es3), single shot and profile of $9 \mathrm{~Hz}$ low frequency shooting is better than that of the $30 \mathrm{~Hz}$ high frequency shooting. This suggests that the penetration ability of volcanic of the low-frequency signal is stronger than that of the high-frequency signal. Therefore, low-frequency vibroseis is chosen, the low frequency information of low frequency is rich, which will improve the ability of penetrating volcanic, meanwhile improve the form of correlative wavelet, extend the spectrum of seismic data, and improve resolution of the seismic data, which become an important tool to break bottlenecks of the volcanic.

\subsection{Bin Size}

Small bin size is not only advantageous to improve the lateral resolution, but also advantageous to improve the 


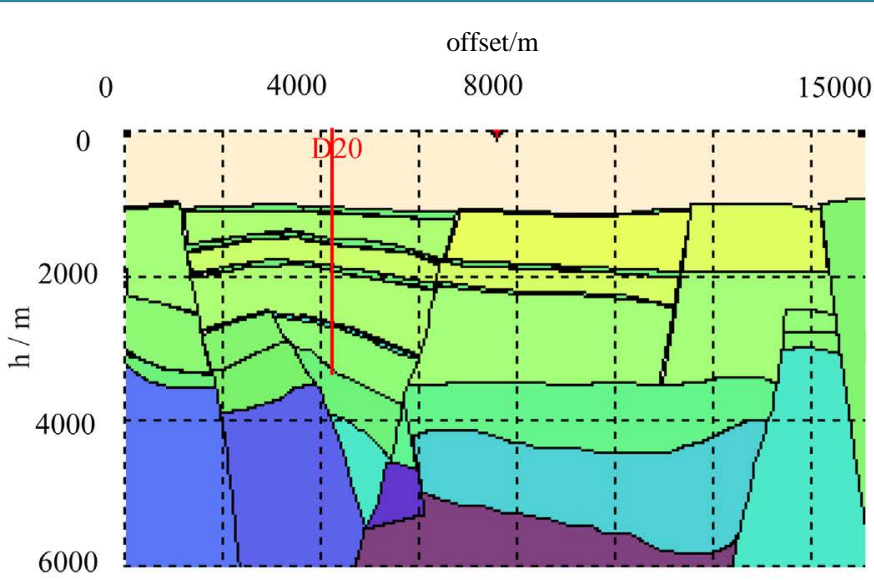

(a)

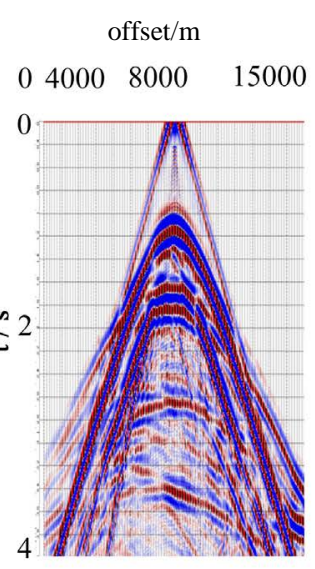

(b)
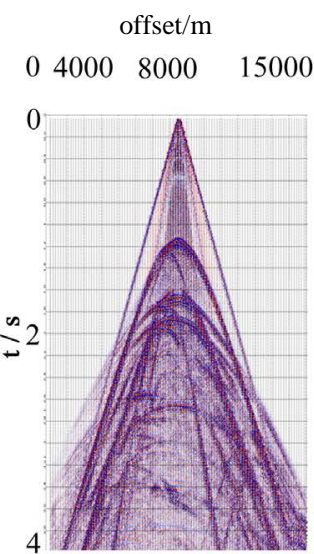

(c)

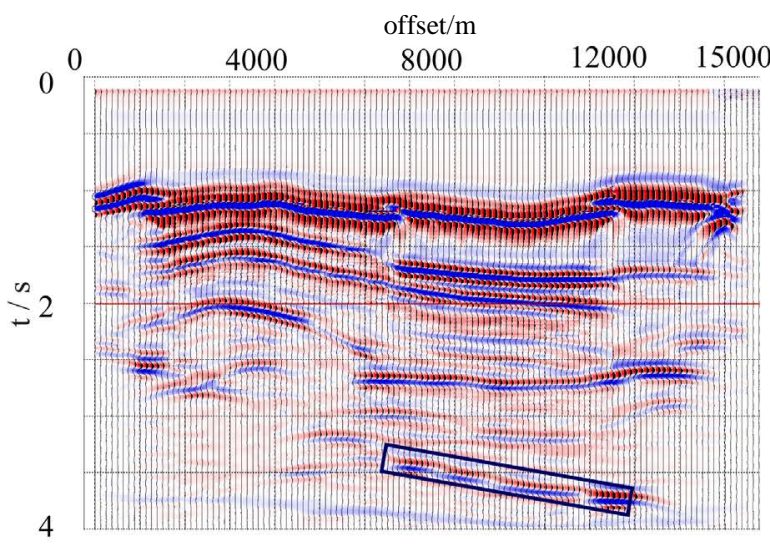

(d)

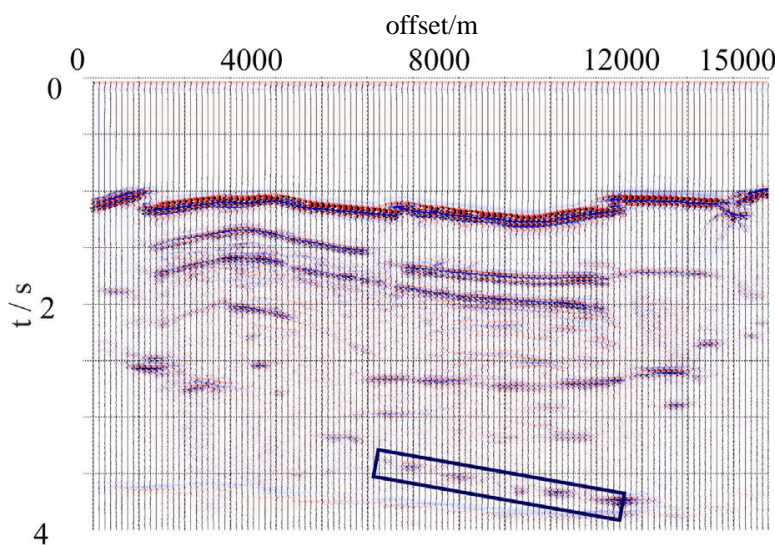

(e)

Figure 1. Seismic geological model through well D20 (a); Forward modeling single shot with dominant frequency $9 \mathrm{~Hz}$ (b); Forward modeling single shot with dominant frequency $30 \mathrm{~Hz}$ (c); PSTM imaging profile with dominant frequency $9 \mathrm{~Hz}$ (d) and PSTM imaging profile with dominant frequency $30 \mathrm{~Hz}(\mathrm{e})$.

vertical resolution [15]; small bin is good for small target geologic body imaging (such as minor fault); small bin size can reduce aliasing and migration noise, which is helpful to improve the quality of the migration data.

Conventional seismic acquisition bin size must meet the requirements of the highest no-aliasing frequency, lateral resolution and migration homing. While high density acquisition bin size should not only have the sufficient sampling of the effective signal, the sampling of the surface waves and shallow refraction cannot pollute the effective signal and can weaken migration noise [16]. In general, the bin size considering the pollution-free sampling is smaller than that of the conventional considering no-aliasing frequency and the lateral resolution.

Usually FK spectrum of single shots with different trace interval are been used to judge whether the regular noises sampling have achieved not pollute effective signal. Figure 2 is FK spectrum of different single shots with different trace interval in HX area. For single shot with trace interval $25 \mathrm{~m}$, noise wave has not pollute effective signal which frequency less than $75 \mathrm{~Hz}$ (the biggest frequency of effective signal of the target stratum is $70 \mathrm{~Hz}$ ). Interference wave realizes non-pollution sampling, which can protect the effective signals of $75 \mathrm{~Hz}$ pollution-free sampling. For single shot with trace interval $50 \mathrm{~m}$, the noise folding frequency and the effective signal are aliasing, which against noise suppression and can only protect the effective signals of $37 \mathrm{~Hz}$ pollution-free sampling. For single shot with trace interval $75 \mathrm{~m}$, the noise folding frequency and the effective signal are aliasing more severe. To sum up, for trace interval $25 \mathrm{~m}$, which can achieve not to pollute effective signal, which can avoid the noise caused by the insufficient sampling density spatial aliasing and avoid subsequent noise suppression hidden trouble. Also, it is unnecessary to narrow the trace interval and constantly increase meaningless exploration cost. 


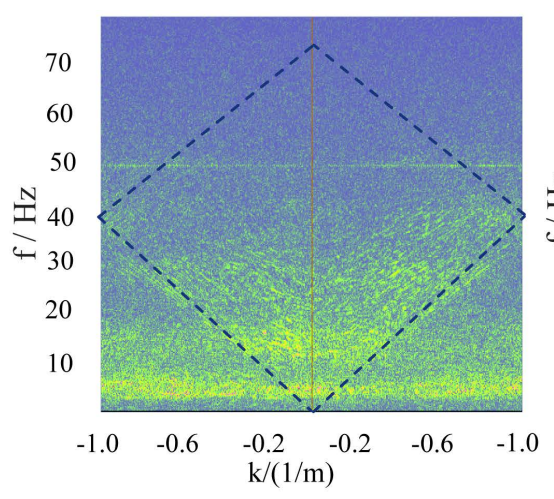

(a)

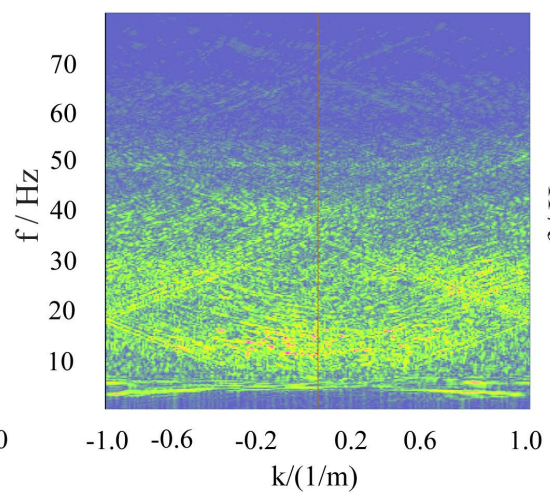

(b)

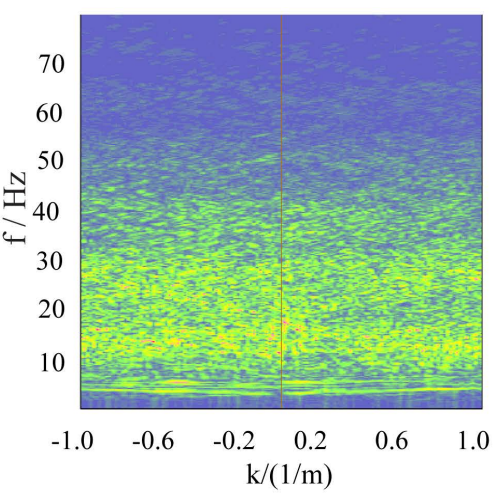

(c)

Figure 2. FK spectrum of single shot records of trace interval $25 \mathrm{~m}$ (a), Trace interval $50 \mathrm{~m}$ (b); Trace interval $75 \mathrm{~m}$ (c).

\subsection{Fold}

Folds can determine the ability of noise attenuation. N Folds can make random noise attenuation to the original $\sqrt{N}$ after stack. Because the volcanic underlying strata noise energy is strong and the effective reflection signal is weak, high folds must be used, which can improve the signal-to-noise ratio of the volcanic development zone. Signal-to-noise ratio is the basis of the resolution. The signal-to-noise of different frequency band are different, usually the higher the frequency, the lower the signal-to-noise ratio, high folds is the best means to improve the signal-to-noise ratio of the high frequency band. Through high density spatial sampling to improve folds. Through increasing the receiving line number to increase the lateral folds, raising the shot and receiver density to increase vertical folds, both can promote 3D exploration of ultra-high folds and improve the signal-to-noise ratio of high frequency band, expand the effective frequency band signal and achieve high resolution exploration [17].

For the 2D wide line research data (total folds is 720 times, excitation mode is dynamite shooting) of the work area, fold degradation processing is conducted, and signal-to-noise ratio of the deep data is analyzed quantitatively. Folds increased from 45 times to 90 times, and then increased to 180 times, the continuity of reflection events of target layer in the stack profile is increasing (Figure 3), the signal-to-noise ratio is increasing (Figure 4). But when the folds increased from 180 times to 360 times, then increased to 450 times, the continuity of reflection events and signal-to-noise ratio increasing extent are small, so the folds greater than 180 times is ok when using dynamite shooting.

According to the signal-to-noise ratio relationship between vibroseis shooting and dynamite shooting of adjacent regions, and through theoretical calculations on the basis of the Formula (1), to achieve the effect of dynamite shooting, the folds of vibroseis shooting should be 2 times of the dynamite shooting (average signal-tonoise ratio of vibroseis shooting single shot is about 0.88 and average signal-to-noise ratio of dynamite shooting single shot is about 1.25), so vibroseis shooting can be used in this area, folds are greater than 360 times and try to be lateral folds and vertical folds evenly.

$$
\text { Fold }_{\text {required }}=\left[\frac{(S / N)_{\text {required }}}{(S / N)_{\text {raw }}}\right]^{2} \text { Fold }_{\text {raw }}
$$

\subsection{Observation Azimuth}

A more full seismic wave field can be got by wide azimuth acquisition, and can better study the amplitude change with offset, azimuth angle and the stratum velocity, which enhances the identification of the fault, fracture and stratigraphic lithology changes. Wide azimuth angle is more easily than the narrow azimuth to step over the surface barrier and remove the influence of underground shadow zone. The multiples attenuation ability of wide azimuth acquisition is better than narrow azimuth acquisition [18]. Wide azimuth (aspect ratio is greater than 0.5) seismic exploration of space imaging resolution is higher than the narrow azimuth [19]. Multiple abroad exploration examples prove that the orthogonal wide azimuth acquisition [20] and full azimuth double 


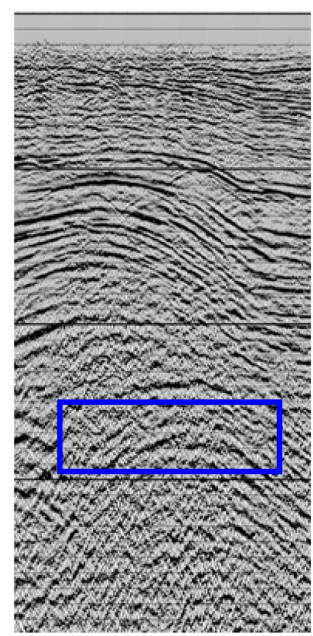

(a)

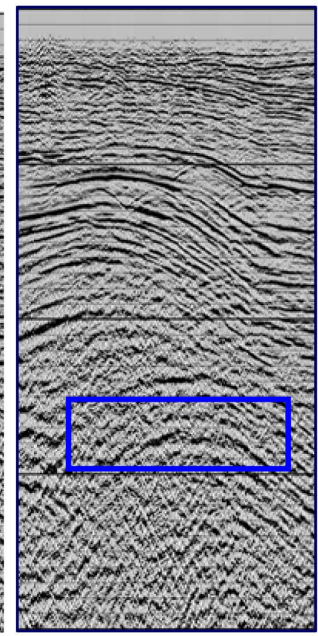

(b)

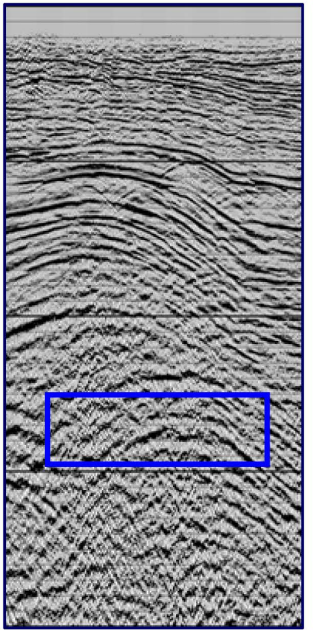

(c)

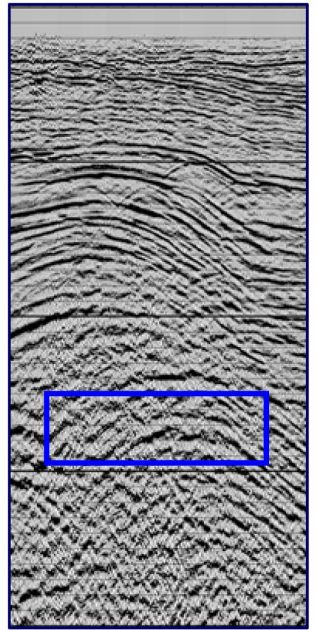

(d)

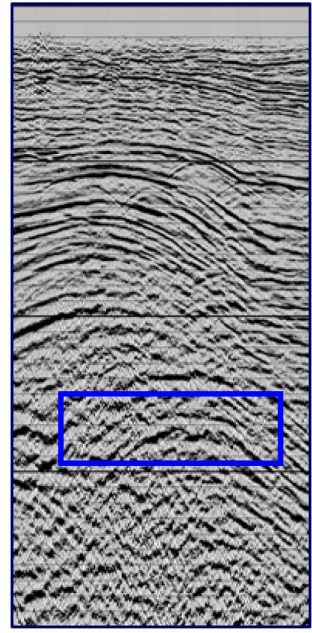

(e)

Figure 3. 2D wide line research data of 45 times (a); 90 times (b); 180 times (c); 360 times (d); 450 times (e) stack profile.

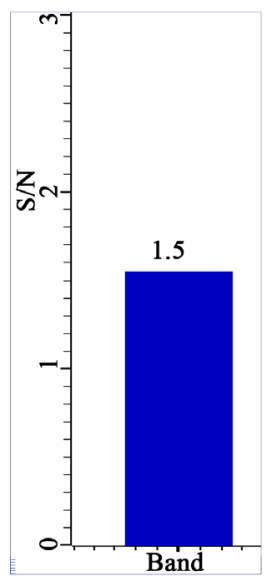

(a)

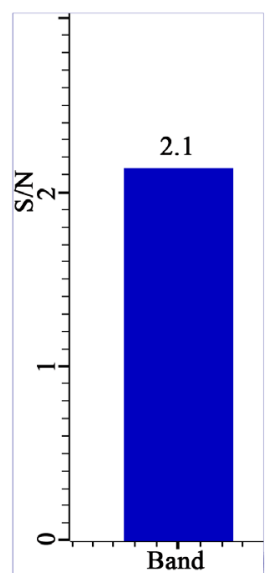

(b)

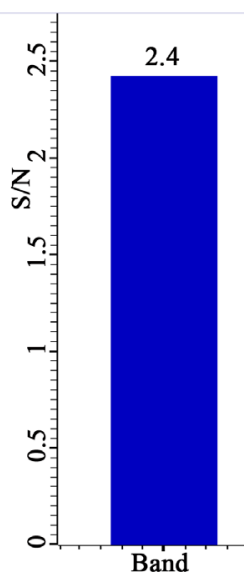

(c)

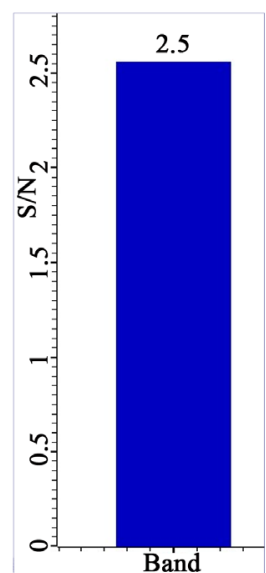

(d)

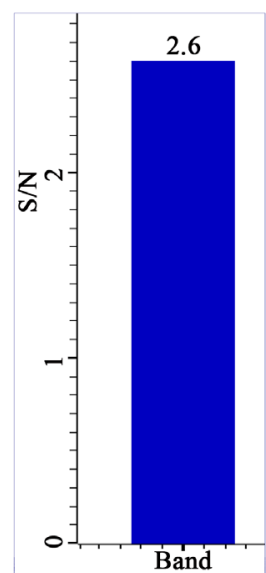

(e)

Figure 4. 2D wide line research data of 45 times (a); 90 times (b); 180 times (c); 360 times (d); 450 times (e) the signal-to-noise ratio of stacked profile in target layer.

helix [21] acquisition is helpful to overcome the shielding effect of salt dome strong reflection and improve the salt dome imaging.

The widely-distributed volcanic of the area has shielding effect on the under layer, which can cause energy shadow. It is beneficial to overcome the influence of shadow zone by the wide azimuth acquisition. On the basis of establishing the 3D model, through the underground geological conditions close to the real 3D model of the wave equation forward modeling and prestack time migration of single shot degenerative processing. This study has analyzed the difference on fault and volcanic underlying imaging between wide and narrow azimuth. HX 3D model contains 18 layers (Figure 5(a)). According to the logging information, vertical from Ed to Es3, there are six sets of volcanic. Volcanic in the middle of the model are thick and it is thin in the boundary. Model of the northwest and southeast are boundary faults. On the basis of the established 3D model, through 3D forward modeling using full azimuth survey (42L4S192T), 3D forward modeling single shots can be got. Through degradation processing, we get different line number PSTM imaging profiles (Figures 5(b)-(e)). By comparison it can be found that survey from $14 \mathrm{~L}$ (aspect ratio is 0.29 ) to $38 \mathrm{~L}$ (aspect ratio is 0.80 ), and then to $42 \mathrm{~L}$ (aspect ratio is 0.9 ). The effect of imaging profiles are improved, namely, fault and volcanic underlying imaging of $38 \mathrm{~L}$ are clearer than that of $14 \mathrm{~L}$, and for $38 \mathrm{~L}$ to $42 \mathrm{~L}$, the imaging effect is not significantly improve. 3D wave equation forward modeling study shows that the aspect ratio should be greater than 0.8 . 


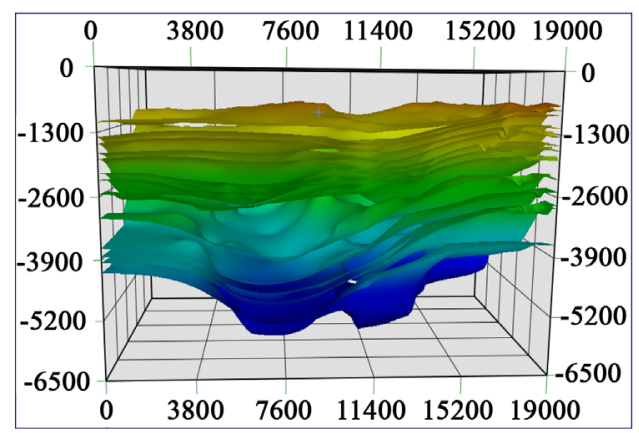

(a)

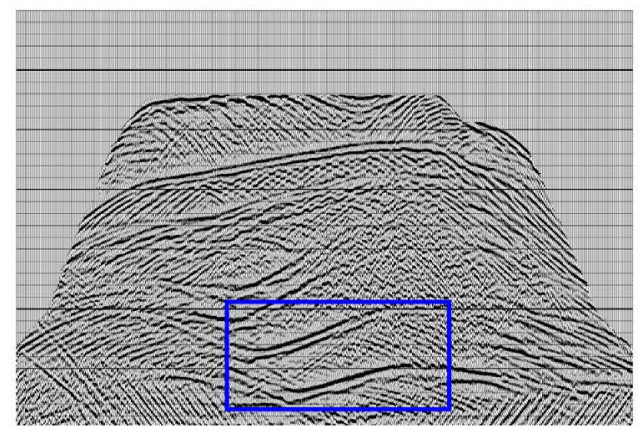

(c)

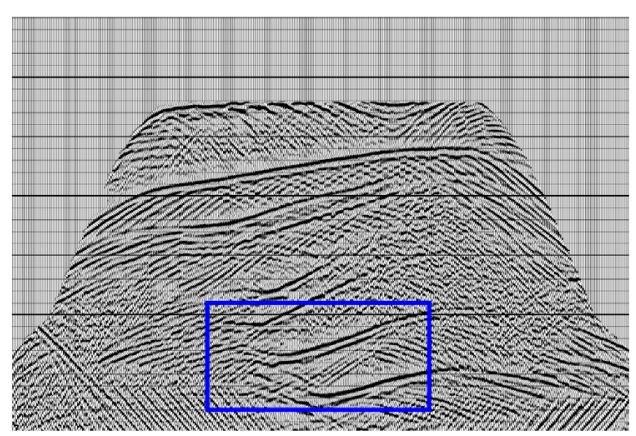

(b)

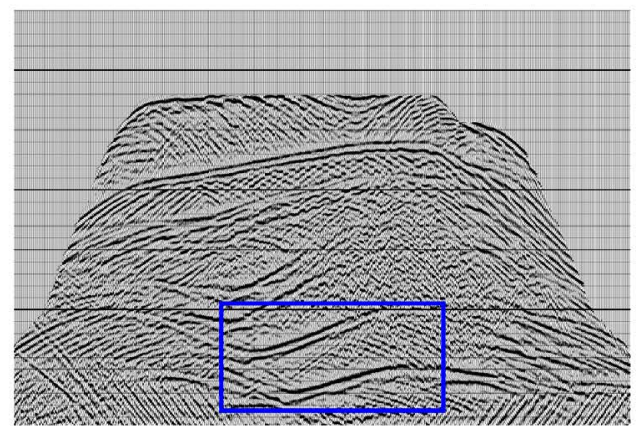

(d)

Figure 5. 3D geological model (a) in HX area and 14 L (b); 38 (c); 42 L (d) PSTM imaging profile of the forward modeling results.

\subsection{Maximum Offset}

The following factors must be mainly considered about the parameter argumentation of the maximum offset: (1) the NMO stretch is less than $12.5 \%$ and velocity analysis accuracy error is less than $6 \%$; (2) to meet the requirements of the multiple removal; (3) 2D wave forward modeling and 3D model theoretical calculation.

The NMO stretch of target layer is less than $12.5 \%$ and the accuracy of velocity analysis error is less than the $6 \%$. The theoretical calculation offset must meet the range: $3484 \mathrm{~m}-6021 \mathrm{~m}$.

In the multiple development area, multiple will seriously affect signal-to-noise ratio, especially be mistaken for effective reflection on single shot, which can lead to misjudgments of data quality. Offset is analyzed considering separate middle multiple wave from deep effective wave. The past CMP gather in the work area is analyzed, to meet the requirement of time difference greater than $0.35 \mathrm{~s}$, the maximum offset should larger than 5000 m (Figure 6).

Wave equation forward modeling analysis is conducted based on the 2D model. The survey is 6987.5-12.525-12.5-6987.5. The forward modeling single shot records and deepest target layer wave field snapshot are analyzed, considering that the maximum offset should be able to capture all the reflection information of the target layer (Es3), the maximum offset should be between 4996 - 5636 m (Figure 7), to meet the requirements of prestack migration processing.

In the present acquisition design, it is often through several argument points to prove the acquisition parameters. From Figure 8(a), buried depth of the target layer (Es3) can vary more than one thousand meters. Analyzing the several argument points to prove acquisition parameters can not well reflect the real structure of the whole exploration area. So for the complex fault zone, on the basis of the established 3D model, according to the target layer depth, the maximum offset analysis of the target layer in the whole area is carry out, the maximum offset for Es3 should be between 4996 - 5882 m (Figure 8(b)).

Summarize the above analysis, the offset should be about $5800 \mathrm{~m}$.

Through the above analysis for main acquisition parameters and according to the sampling principle [22] [23] "fully, evenly, symmetrical, wave field continuity", and keep the wave field primitive and rich, and try to avoid the spatial aliasing and migration noise, which ultimately determine the wide azimuth high-density 3D seismic 

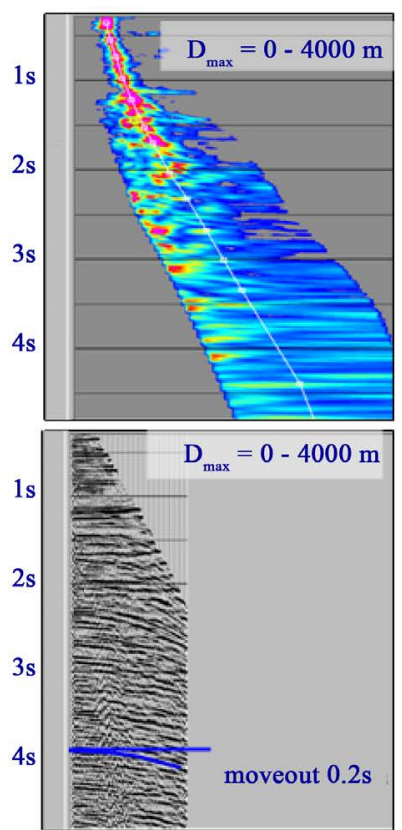
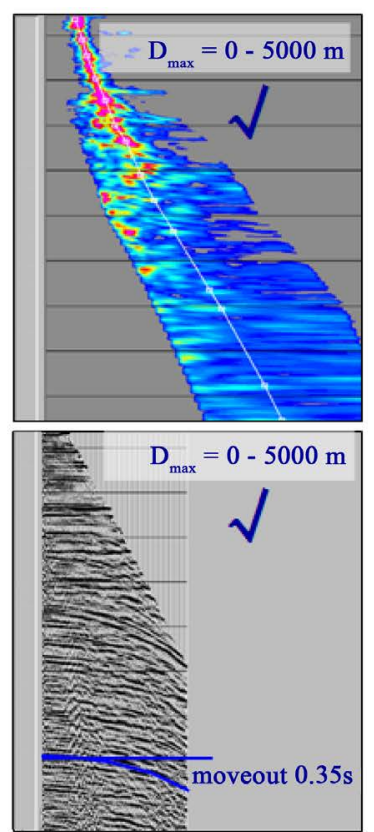
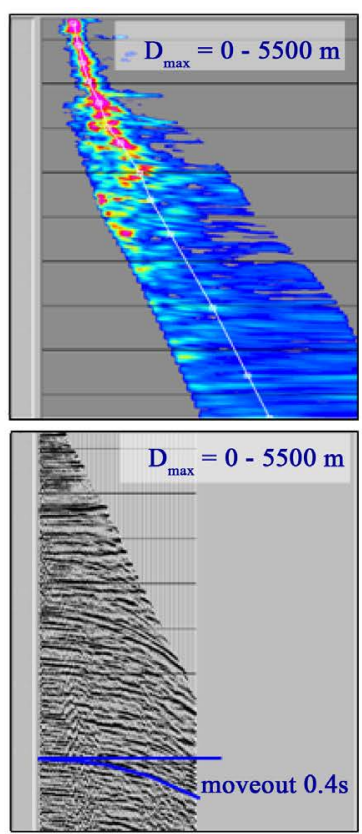
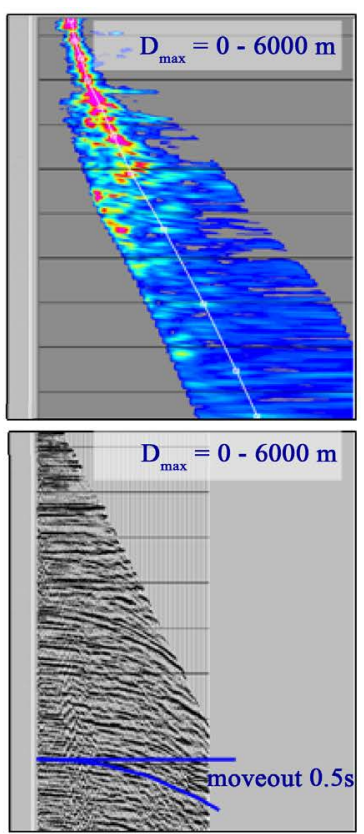

Figure 6. The contrast analysis of time difference between different maximum offset multiple wave.

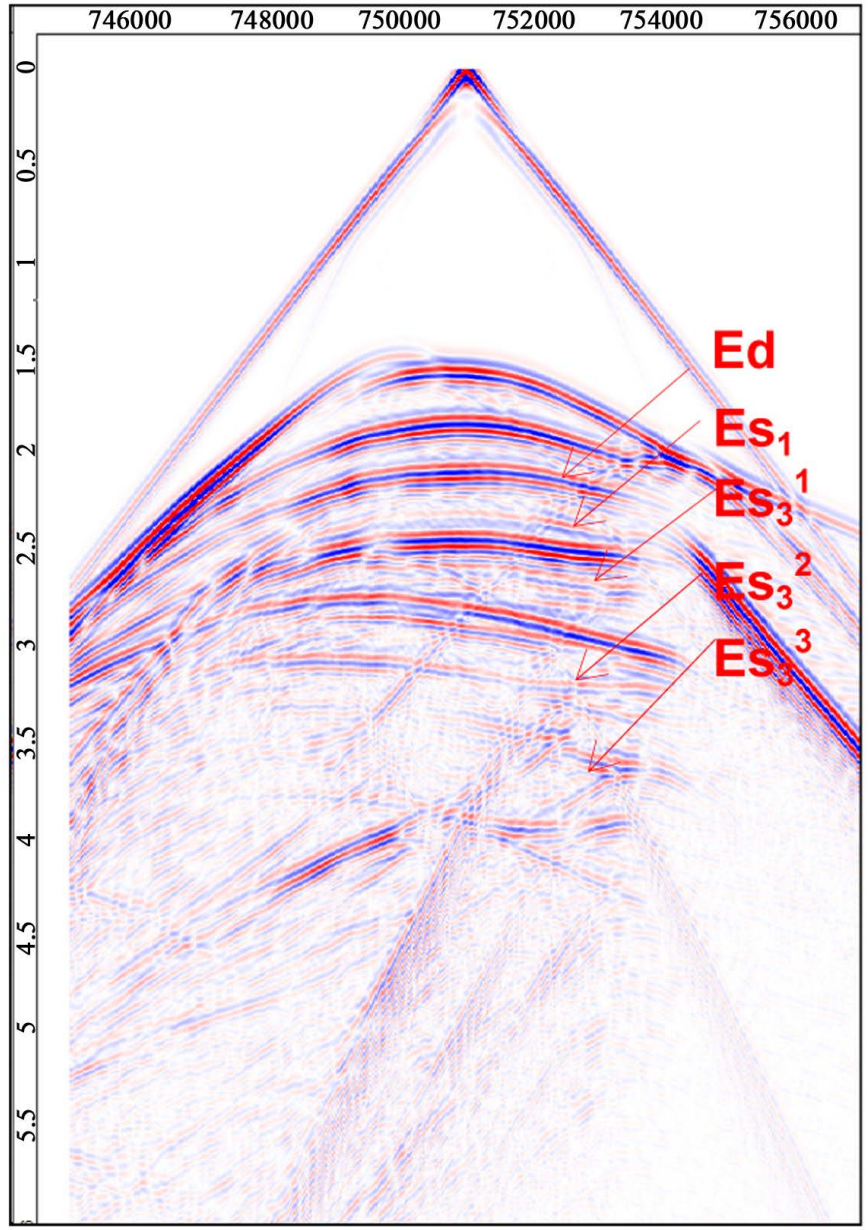

(a) 


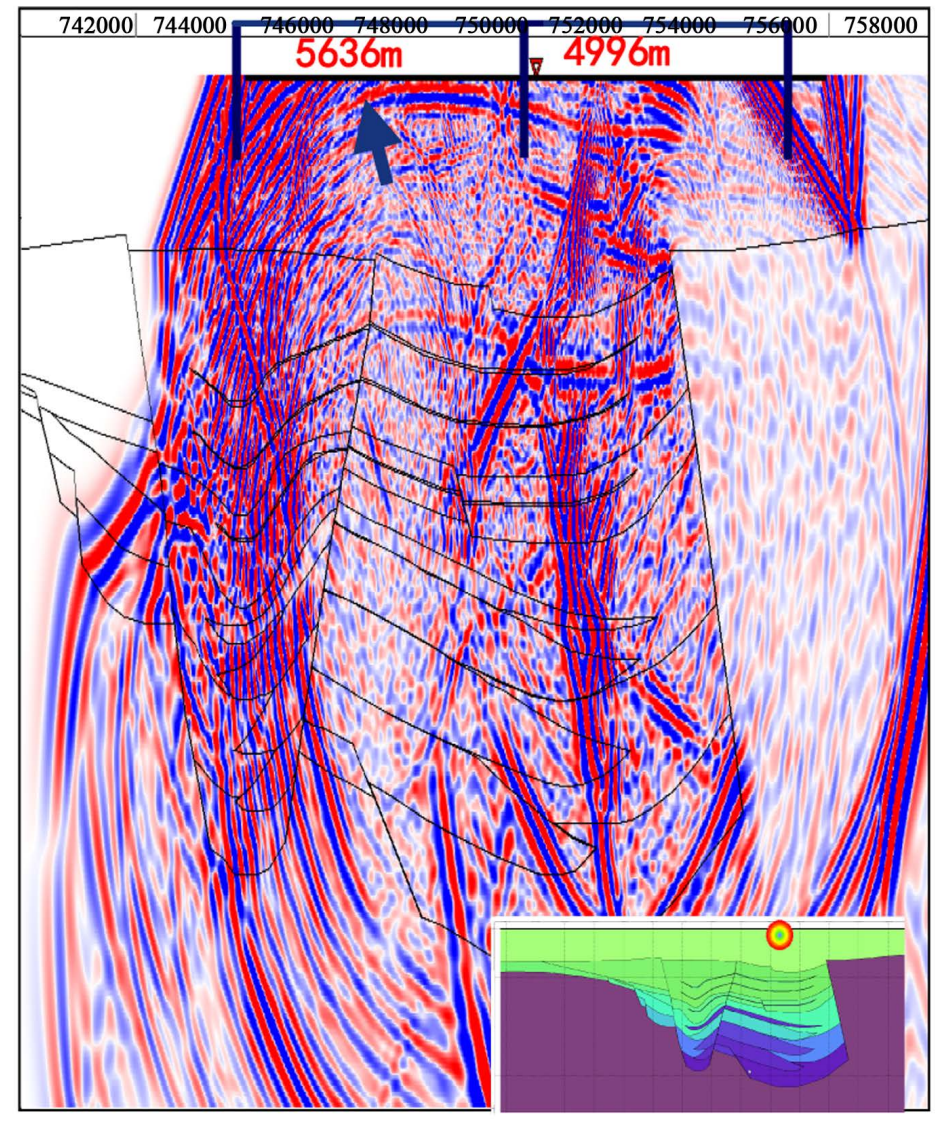

(b)

Figure 7. 2D forward modeling single shot (a) and the wave field snapshots (b).

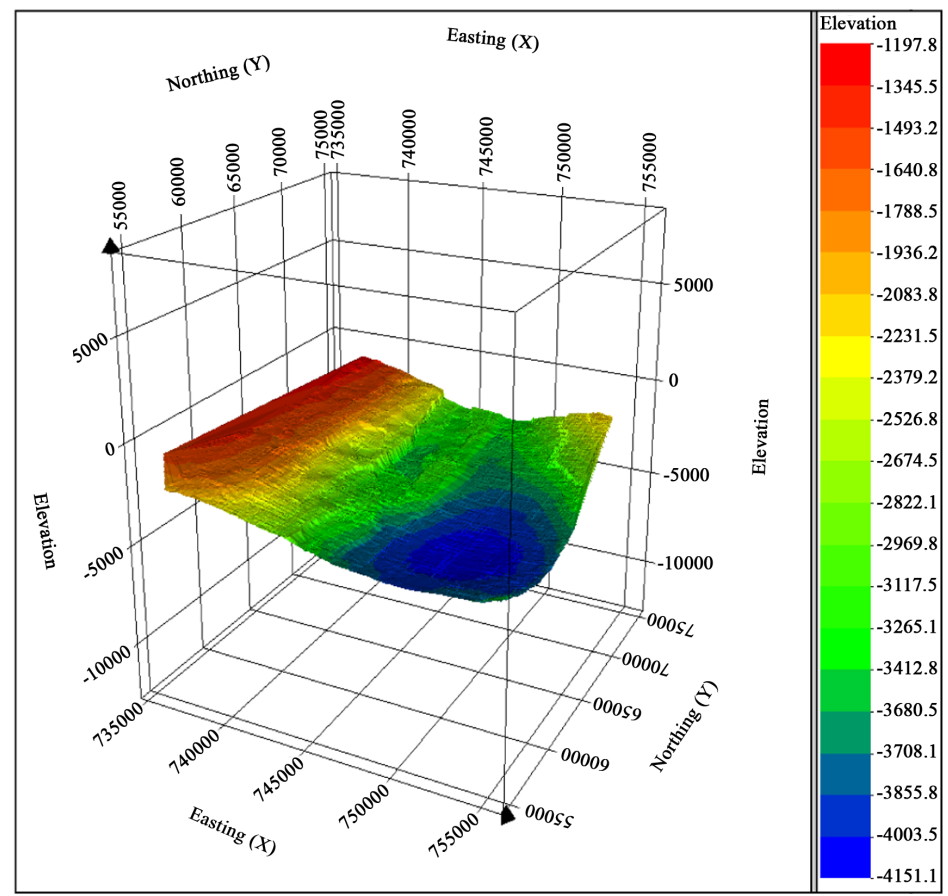

(a) 


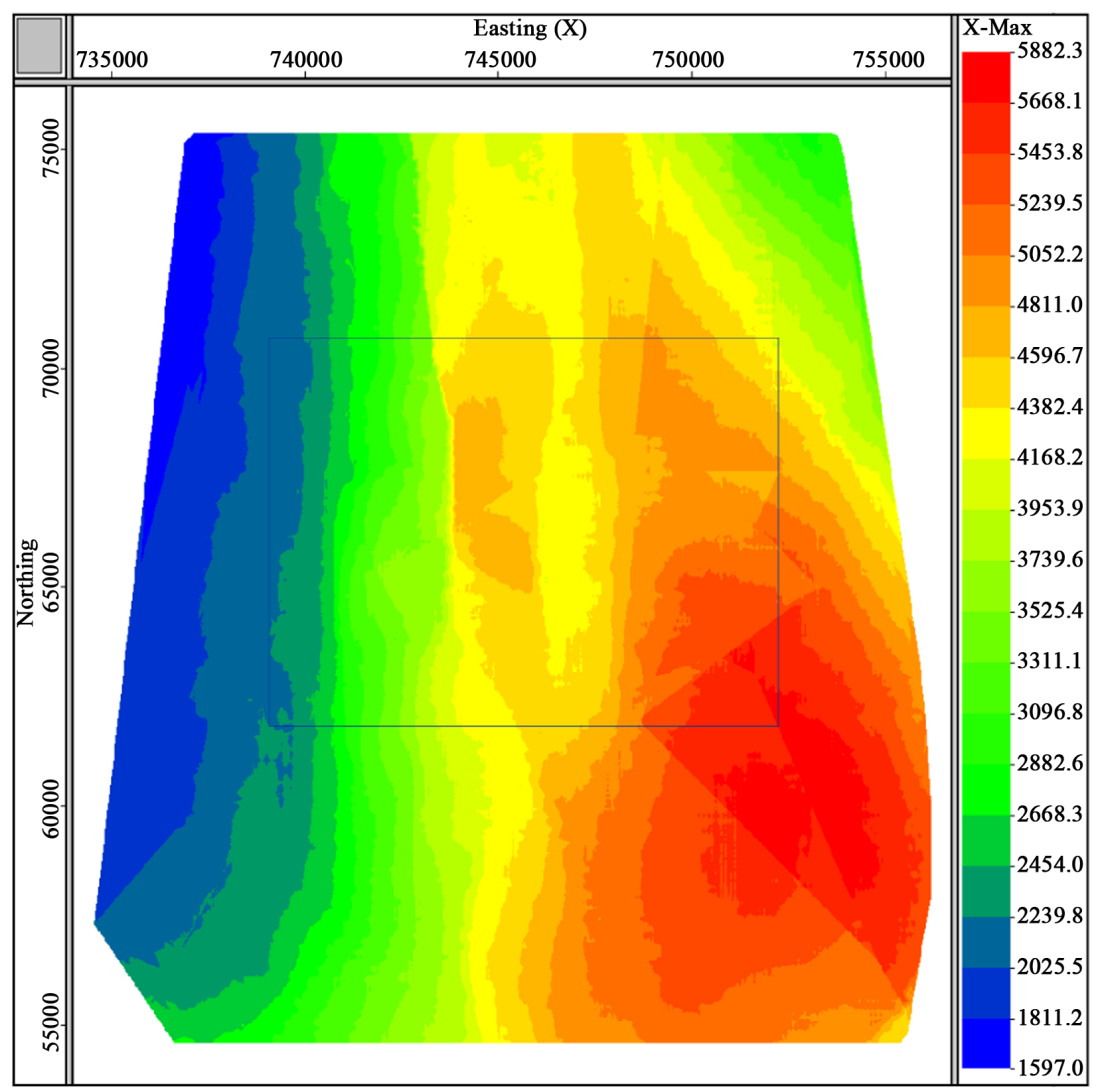

(b)

Figure 8. 3D model in Es3 (a) and the calculation results of maximum offset (b).
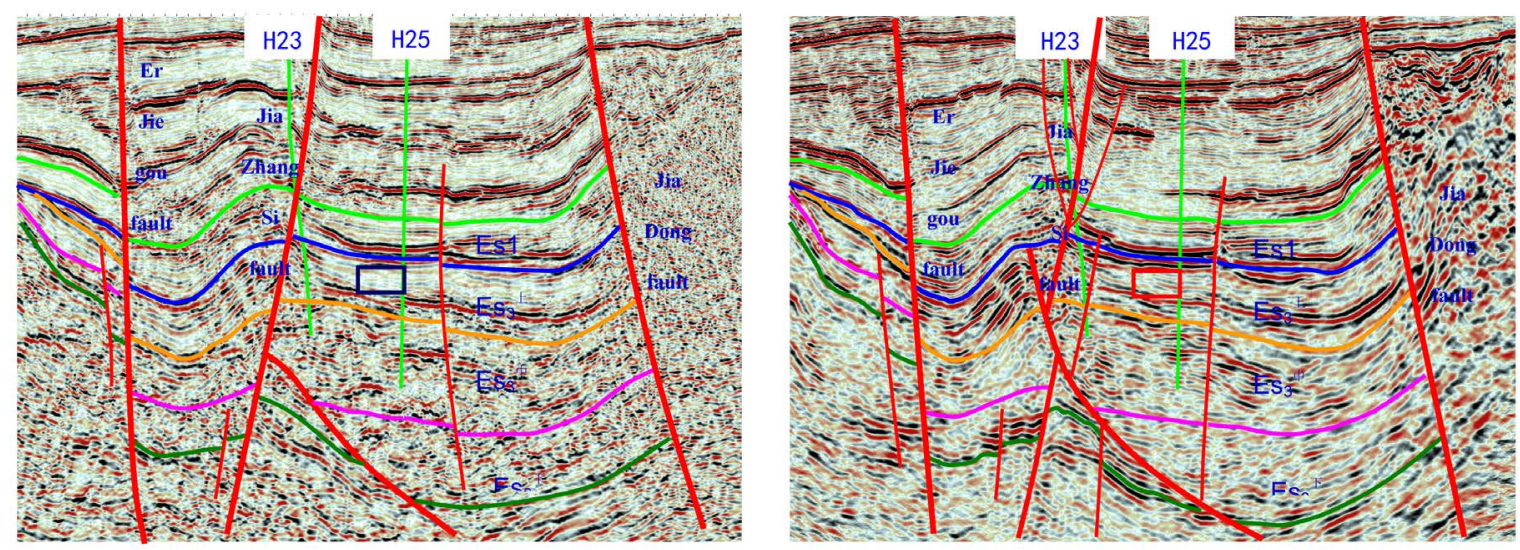

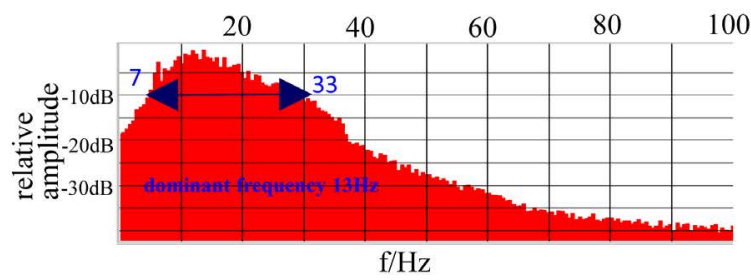

(a)

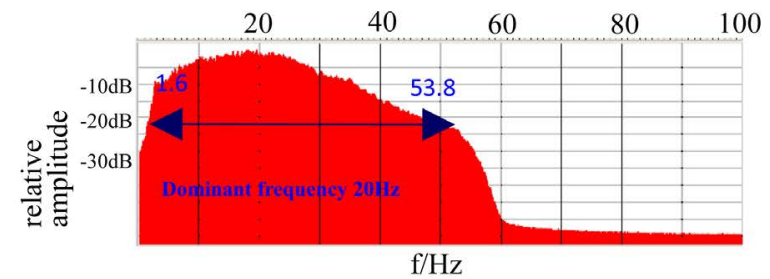

(b)

Figure 9. Conventional 3D prestack migration and spectrum (a) and "Two Wide and One High" 3D prestack migration spectrum (b). 
acquisition survey, observation method: $36 \mathrm{~L} \times 4 \mathrm{~S} \times 352 \mathrm{~T}$ orthogonal; Bin size: $12.5 \mathrm{~m} \times 12.5 \mathrm{~m}$; Fold number: 396 times; Total trace number: 12,672; Trace interval/shot interval: $25 \mathrm{~m} / 25 \mathrm{~m}$; Receiving line interval/Shot line interval: $200 \mathrm{~m} / 200 \mathrm{~m}$; the maximum offset: $5668 \mathrm{~m}$; aspect ratio: 0.82 (achieve full azimuth for $4000 \mathrm{~m}$ target layer).

\section{Effect of the Seismic Data}

"Two Wide and One High" scheme is used in the HX 3D acquisition, processing and interpretation research have been conducted. From the migration profile and spectrum analysis (Figure 9), every reflection event is rich in the new high density profile, the low frequency information is rich, low-frequency expanding $5 \mathrm{~Hz}$ compared the old data, high-frequency expanding $20 \mathrm{~Hz}$, octave increased significantly. The signal-to-noise ratio of new acquisition high-density 3D data is high, and the resolution is improved obviously. For the new acquisition data, it is blank weak reflection on west side of Jia Zhang Si fault, and is disorder reflection on the east side of Jia Zhang Si fault, further confirmed on both sides of the fault have the characteristics of lithology mutation. For the old data, it is disorder reflection on both sides of Jia Zhang Si fault, which does not reflect lithology mutations.

From horizon coherence cube slices, for the new acquisition data, the Jia Zhang Si fault and its associated fault recognition ability improve significantly, Jia Zhang Si fault distribution is clear, The Jia Zhang Si fault and its derived tuned fault formed a series of structural trap. Jia Zhang Si fault has the function of connecting oil source, the structural traps formed by Jia Zhang Si fault and its derived tuned fault is favorable for hydrocarbon accumulation. Through the fine structure interpretation, newly discovered six EW-trending faults (blue dashed line as shown in Figure 10(b), and carry out the favorable structure $3.8 \mathrm{~km}^{2}$. H28 on both sides and H26 in northern area can be used as the next important exploration.

\section{Conclusions}

By studying and summarizing survey parameters prove and processing and interpretation result of this volcanic development area, there are the following conclusions:

1) Compared with the high frequency wavelet, low frequency wavelet can penetrate volcanic shield more

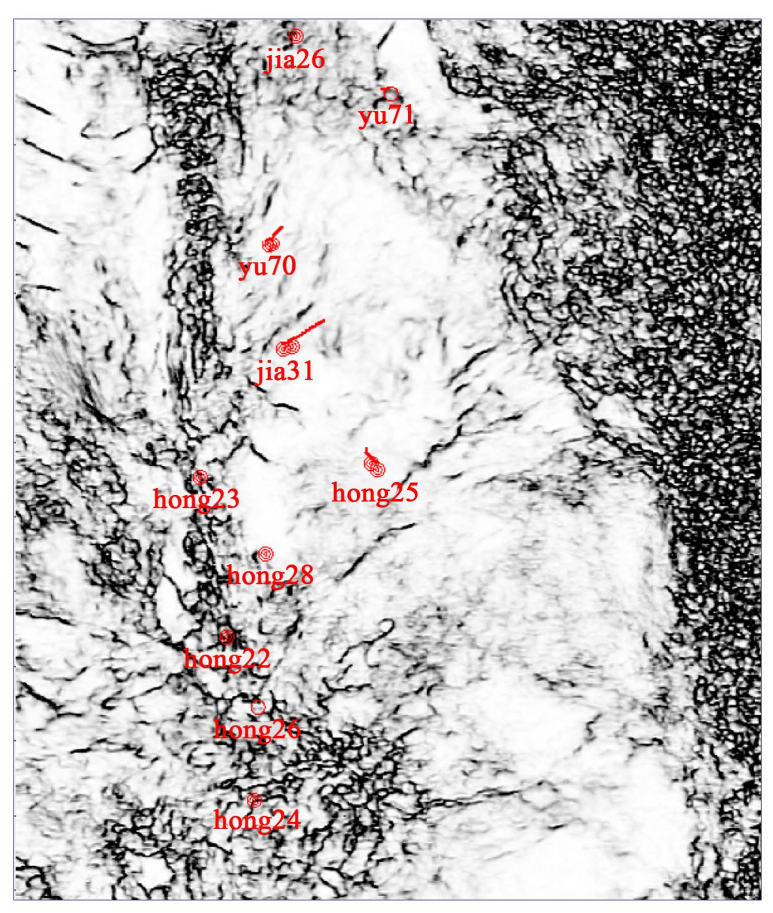

(a)

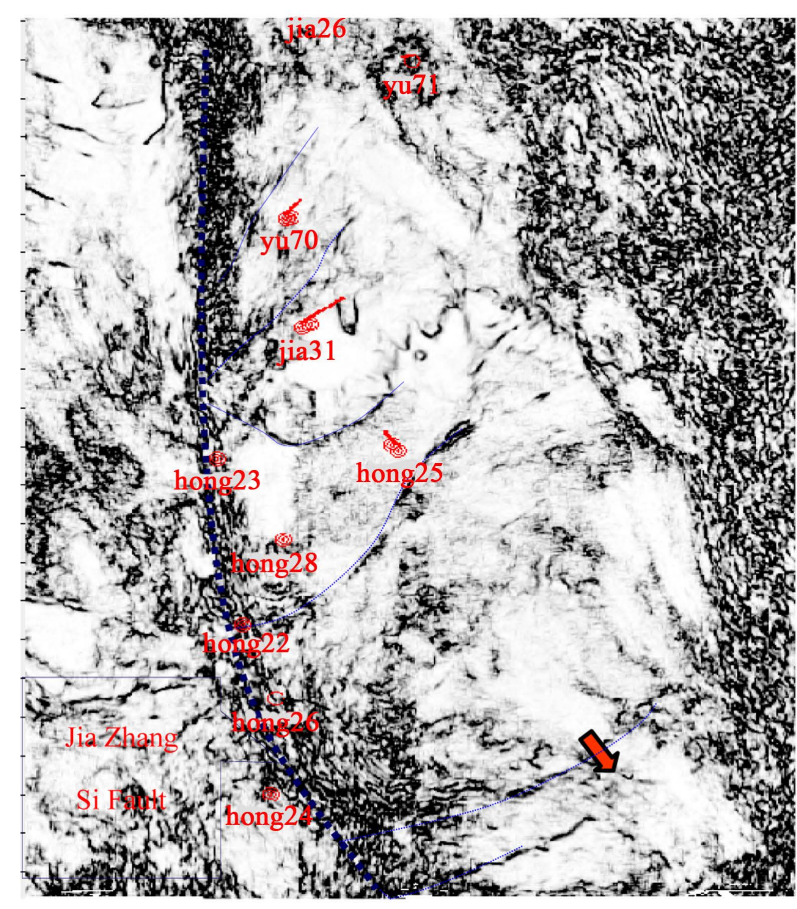

(b)

Figure 10. Coherence cube slice of conventional 3D prestack time migration data (a) and Coherence cube slice of "Two Wide and One High” 3D prestack time migration data (b). 
effectively. Using low frequency source can expand the low frequency component of the volcanic underlying strata effectively.

2) Based on the survey of small bin, high fold and wide azimuth, effectively improves the signal-to-noise ratio and resolution of the volcanic area data, and improves the precision of fault imaging. The information of Es3 is rich and continuity in axis improves a lot.

3) It is easy to distinguish effective wave from multiple wave with enough spread length, which can ensure the follow-up processing data accurately.

To sum up, "Two Wide and One High" scheme can effectively solve the problem of the volcanic imaging, which can provide technical reference for the similar volcanic development area in the future seismic acquisition design.

\section{References}

[1] Fliedner, M.M. and White, R.S. (1999) Using Wide-Angle Seismic Data for Basalt and Sub-Basalt Imaging. Expanded Abstracts of 61th Annual Internat SEG Mtg.

[2] Haugen, G.U., Toil, S. and Yu, F.H. (1998) Can Seismic Wide-Aperture Data Image Beneath Basalt. Expanded Abstracts of 60th Annual Internat SEG Mtg, 90-93.

[3] Fruehn, J., White, R.S., Fliedner, M., et al. (1999) Large-Aperture Seismic: Imaging Beneath High-Velocity Strata. World Oil, 220, 109-113.

[4] Makris, J., Rihm, R. and Egloff, F. (1999) WARRP (Wide Aperture Reflection and Refraction Profiling): The Principle of Successful Data Acquisition Where Conventional Seismic Fails. Expanded Abstracts of 61th Annual Internat SEG Mtg, 989-992.

[5] Purnellg, G.W. (1992) Imaging Beneath a High Velocity Layer Using Converted Waves. Geophysics, 57, $1444-1452$. http://dx.doi.org/10.1190/1.1443212

[6] Moshe, R.H. and Ben-Avraham, Z. (2003) A Case Study of Sub-Basalt Imaging in Land Region Covered with Basalt Flows. Geophysical Prospecting, 51, 247-260. http://dx.doi.org/10.1046/j.1365-2478.2003.00368.x

[7] Ziolkowski, A., Hanssen, P., Gatliff, R., et al. (2003) Use of Low Frequencies for Sub-Basalt Imaging. Geophysical Prospecting, 51, 169-182. http://dx.doi.org/10.1046/j.1365-2478.2003.00363.x

[8] Hu, Z.P., Guan, L.P., Gu, L.X., et al. (2004) Wide Angle Seismic Wave Field Analysis and Imaging Method below the High Velocity Shield Layers. Chinese Journal of Geophysics, 47, 88-94. http://dx.doi.org/10.1002/cjg2.459

[9] An, X.Y., Li, L.W. and Yu, P.F. (2004) Seismic Data Acquisition in the Igneous Rock Area of Erlian Basin. GPP, 43, 171-175.

[10] Zhao, J.Z., Zhang, J.D., Gu, Y.M., et al. (2010) Research of 3D Seismic Data Acquisition Techniques in Igneous Rock Area, Hainan. China Petroleum Exploration, 4, 57-64.

[11] She, D.P., Guan, L.P., Xu, Y., et al. (2007) Applying of Low-Frequency Signal to Improve Imaging Quality below Shielding High-Velocity Basalt. OGP, 42, 564-567.

[12] She, D.P., Wu, J.M., Li, P., et al. (2006) Application of Low Frequency Signal to Imaging Anhydrite Based on Modeling. CPP, 45, 234-238.

[13] Zhang, G.D. (2013) Forward Modeling and Geometry Optimization of the Igneous Rocks Structure within Huimin Depression. Progress in Geophys, 28, 3098-3105.

[14] Zhao, B.L., Wang, X.S., Dong, S.T., et al. (2014) Geophysical Prospecting Requirements and Development Direction in Bohai Bay Basin. OGP, 49, 394-409.

[15] Xiong, J.L., Yue, Y., Yang, Y., et al. (2006) Relationship between Bin Size and Inline Resolution. OGP, 41, $489-491$.

[16] Wang, M.S., Hu, Y.G., Wang, Q.C., et al. (2009) Parameter Selection in High-Density Seismic Acquisition. PEG, 32, 404-408.

[17] Zhao, H.X., Jin, Z.G., Zhang, Y.S., Hu, C.J. and Liu, C.X. (2007) Fold Selection in the High Density Sampling Seismic Acquisition. Natural Gas Industry, 27, 68-69.

[18] Cordsen, A. and Galbraith, M. (2002) Narrow- versus Wide-Azimuth Land 3D Surveys. The Leading Edge, 21, 764770. http://dx.doi.org/10.1190/1.1503181

[19] Ling, Y., Gao, J., Sun, D.S. and Wu, L. (2005) Analysis and Appreciation of Wide/Narrow Azimuth Exploration Cases (1). Oil Geophysical Prospecting, 40, 305-308.

[20] Baldock, S., Tang, C.R., Beck, B., Gao, W., Cai, J. and Hightower, S. (2012) Orthogonal Wide Azimuth Surveys: Acquisition and Imaging. First Break, 30, 35-41. http://dx.doi.org/10.3997/1365-2397.2012012 
[21] Brice, T. (2011) Designing, Acquiring and Processing a Multivessel Coil Survey in the Gulf of Mexico. SEG Technical Program Expanded Abstract 2011, 92-96. http://dx.doi.org/10.1190/1.3628225

[22] Cooper, N. (2004) A World of Reality-Designing Land 3D Programs for Signal, Noise, and Prestack Migration: Part 1 of a 2-Part Tutorial. The Leading Edge, 23, 1007-1014. http://dx.doi.org/10.1190/1.1813357

[23] Cooper, N. (2004) A World of Reality-Designing Land 3D Programs for Signal, Noise, and Prestack Migration: Part 2 of a 2-Part Tutorial. The Leading Edge, 23, 1230-1235. http://dx.doi.org/10.1190/leedff.23.1230_1

\section{Submit or recommend next manuscript to SCIRP and we will provide best service for you:}

Accepting pre-submission inquiries through Email, Facebook, Linkedin, Twitter, etc A wide selection of journals (inclusive of 9 subjects, more than 200 journals)

Providing a 24-hour high-quality service

User-friendly online submission system

Fair and swift peer-review system

Efficient typesetting and proofreading procedure

Display of the result of downloads and visits, as well as the number of cited articles

Maximum dissemination of your research work

Submit your manuscript at: http://papersubmission.scirp.org/ 